\title{
Nuclear astrophysics studies at NIPNE
}

\author{
Livius Trache
}

Horia Hulubei Institute for Physics and Nuclear Engineering, Bucharest-Magurele, Romania

\begin{abstract}
I will present results of doing nuclear astrophysics research at the National Institute for Physics and Nuclear Engineering, Bucharest-Magurele in the last 2-3 years. Own Nuclear Astrophysics Group (NAG) is focused on the basic types of experiments:

Direct measurements at low and very low energies with ion or alpha beams from the local $3 \mathrm{MV}$ tandetron accelerator. We concentrate on activation measurements. The use of the ultra-low background laboratory in a salt mine at Slanic-Prahova, about 120 $\mathrm{km}$ away and of a beta-gamma coincidence unit at home is providing competitive sensitivity.

Indirect measurements done with beams at international facilities with radioactive beams: TAMU and RIBF RIKEN.

With help from colleagues, I will mention some theory advances, too.
\end{abstract}

\section{Introduction}

I will start with thanking, twice, the organizers: for inviting me and for suggesting the title and subject of this talk. The charge was to present the activities in nuclear physics for astrophysics at my home institution, the National Institute for Physics and Nuclear Engineering (NIPNE) in Bucharest-Magurele, also known as IFIN-HH from its Romanian name. This charge is similar with the one given me two editions ago, and I was glad to oblige. For some reasons, in particular the loss of manpower, the experimental activities were concentrated in one group, the Nuclear Astrophysics Group (NAG), which at some point had 7 members. I will, therefore, concentrate in presenting its work, and only at the end list in a few sentences the progress in theory by other groups or scientists of NIPNE. While the main lines of work are the same as presented earlier [1], we are now at the point where we went from plans and proposals to results. One further development is that some of the young members diversify by working in groups at foreign institutes or universities. I will, however, talk only about their work derived from specific plans in NIPNE.

We continued the two main types of nuclear astrophysics experiments:

- direct measurements, done at home, using the $3 \mathrm{MV}$ tandetron, mainly using activation techniques. These use low-energy stable beams, an ultra-low background laboratory and a new beta-gamma coincidence setup. The ${ }^{12} \mathrm{C}+{ }^{12} \mathrm{C}$ fusion reaction was the focus of one long set of measurements, $(\alpha, \gamma)$ reactions in the rp-region of nuclei was another.

- indirect methods, using rare ion beams or exotic nuclei produced at international facilities.

I will treat each of these in the following sections.

In the end I will briefly state the interest in developing theory support tools for our indirect methods and the coupling of nuclear structure theory to nuclear processes that take place in stars.

\section{Experiments with low-energy stable beams}

In the last years we studied the possibility to do direct measurements for nuclear astrophysics in IFIN-HH. (as for example $(p, \gamma),(\alpha, \gamma)$ or ion-ion fusion), We realized that we can be competitive using alpha and light ion beams, but not using proton beams. The small $3 \mathrm{MV}$ tandetron accelerator can deliver stable and relatively intense beams of light 
ions and alphas. A list is in [1] and the complete list of beams available can be found at http://www.nipne.ro/research/departments/dfn.php under "Accelerators/ 3 MV tandetron". These two types of beams were used in our experiments.

\subsection{The study of ion-ion fusion}

The one experiment we worked most and with results was the study of the ${ }^{13} \mathrm{C}+{ }^{12} \mathrm{C}$ reaction. While this is not a reaction of importance in nuclear astrophysics, we and our collaborators from the Institute of Modern Physics, Lanzhou, China, claim that its study can help to understand the fusion mechanism for the ${ }^{12} \mathrm{C}+{ }^{12} \mathrm{C}$ reaction at very low sub-barrier energies. The latter reaction is very important in stars, and reliable experimental information is not available in the Gamow window, despite many attempts using direct [2] and references therein, or indirect methods [3]. We claim that our measurements with ${ }^{13} \mathrm{C}$ beams at energies deep in the Gamow window help to understand the fusion mechanism and gives an upper limit for the fusion cross section of the ${ }^{12} \mathrm{C}+{ }^{12} \mathrm{C}$ reaction. The measurements of the latter are complicated not only by the very small cross sections, but also by the resonances that were found to dominate the region adjacent to that important in astrophysics (see [3] and references therein). The use of the ${ }^{13} \mathrm{C}$ induced fusion is based on the observation that its fusion excitation function is smooth when compared with that of ${ }^{12} \mathrm{C}+{ }^{12} \mathrm{C}$ and has the same trend as it goes down in energy (it touches the top of the resonances). Experimentally, the measurements at very low energies are helped by that that one fusion-evaporation channel ${ }^{13} \mathrm{C}+{ }^{12} \mathrm{C} \rightarrow{ }^{25} \mathrm{Mg}^{*} \rightarrow{ }^{24} \mathrm{Na}^{*}+\mathrm{p}$ leads to a radioactivity which has $\mathrm{T}_{1 / 2}=15.0 \mathrm{~h}$. This enables the use of activation techniques, many times more sensitive at low energies, and the halflife is excellent for using the ultra-low background laboratory IFIN-HH has in the salt mine in Slanic-Prahova, about $120 \mathrm{~km}$ away. The procedure we used and the main characteristics of the facility accelerator + salt mine laboratory are described in a forthcoming publication [4].

Summarizing, the pros of this accelerator + salt mine facility are:

- a small but stable accelerator with relatively high currents, including for light ions;

- a laboratory with ultra-low gamma-ray background in a salt mine for de-activation measurements with high resolution and efficient HPGe detectors;

- the ability to determine absolute values for the cross sections measured - we have an utility (GammaSpec), which is certified and cross calibrated internationally.

The limitation stems from the distance between the accelerator and the salt mine, which makes it inefficient for activities with half-lives shorter than 1-2 hours.

Prompt gamma-rays were measured with HPGe detectors, and gammas from de-activation were measured in three setups, carefully calibrated and intercompared. With our measurements at energies $\mathrm{E}_{\mathrm{cm}}=5.5-2.3 \mathrm{MeV}$ we got in the region of energies corresponding to the Gamow window and could measure a cross section of 130(30) pb at the lowest energy point. This is 100 times lower than the best experiment so far at a surface laboratory. At higher energies we could measure prompt gamma-rays, but at the lowest energies we had to do one day of irradiation, transfer to Slanic in $2.5 \mathrm{hrs}$ and about one day de-activation measurement there, during the irradiation of the next target, and so on. For the lowest two energy points, at $\mathrm{E}_{\mathrm{lab}}=4.6$ and $4.8 \mathrm{MeV}$, we irradiated 3 targets each.

The result of the ${ }^{13} \mathrm{C}+{ }^{12} \mathrm{C}$ project is that we disagree with the so-called hindrance model [2] for the fusion of ${ }^{12} \mathrm{C}+{ }^{12} \mathrm{C}$ and extend the astrophysical S-factor down to lower energies in trend with many potential model predictions and in good agreement with the predictions of Caughlan and Fowler [5]. The results were submitted for publication [6].

In continuation of this project we started working on other combinations ion-ion to measure deep sub-barrier fusion. Because activation is the method with best sensitivity, but many residual nuclei in this region do not have lifetimes sufficiently long for efficient transfer to 
the salt mine in Slanic, we designed and made a setup for efficient beta-gamma coincidences that we call BEGA. It was tested with sources, it works, and now we are testing to determine the lower limits of the lifetimes we can access with it located in the accelerator target room.

\subsection{Alpha-beam induced reactions at low energies}

Two reactions were studied with alpha beams of low energy: ${ }^{64} \mathrm{Zn}+\alpha$ and ${ }^{58} \mathrm{Ni}+\alpha$. During experiments natural thick $\mathrm{Zn}$ and Ni targets $(1 \mathrm{~mm})$ were irradiated by alpha beams with energies in the laboratory frame between $5.4-8.0 \mathrm{MeV}$, in steps of 0.2 and $0.25 \mathrm{MeV}$. In the first case the total beam time was $140 \mathrm{~h}$ and the beam current varied from 0.2 to $0.7 \mathrm{~mA}$. For the energy range above we have measured the proton evaporation channel, ${ }^{64} \mathrm{Zn}(\alpha, \mathrm{p}){ }^{67} \mathrm{Ga}$ with a $\mathrm{T}_{1 / 2}=78.28 \mathrm{~h}$, which is one of the three channels that lead to activation. De-activation gamma rays of $\mathrm{E}_{\gamma}=184.6,209.0,300.2$ and $393.5 \mathrm{keV}$ were detected. In this case the sensitivity is increased again by a factor of around 100 compared with previous results [7]. For the second case, the reaction cross sections for the ${ }^{58} \mathrm{Ni}(\alpha, \gamma){ }^{62} \mathrm{Zn}$ and ${ }^{58} \mathrm{Ni}$ $(\alpha, p){ }^{61} \mathrm{Cu}$ channels, with half-lives $\mathrm{T}_{1 / 2}\left({ }^{62} \mathrm{Zn}\right)=9.26 \mathrm{~h}$ and $\mathrm{T}_{1 / 2}\left({ }^{61} \mathrm{Cu}\right)=3.33 \mathrm{~h}$, respectively were determined with a similar sensitivity. In the cases of both $\mathrm{Zn}$ and $\mathrm{Ni}$ targets the transfer to the salt mine laboratory could be used at the lowest energies. The details of these experiments and the results will be the subject of other publications. One example of comparisons of our data with previous experiments is shown in Figure 1 below (A. Chilug et al., to be published). The activation method (labelled "IFIN decay") is significantly more sensitive than the prompt, in-beam measurements and covers 6-7 orders of magnitude.

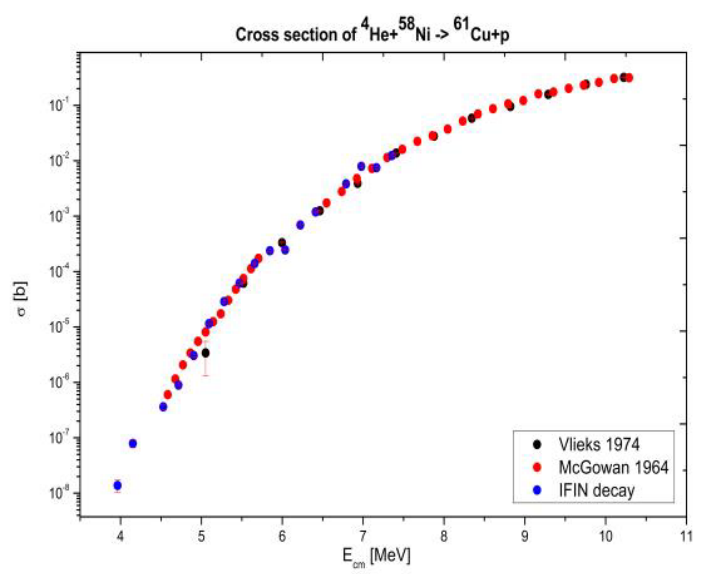

Figure 1. Comparison between the results obtained in IFIN and in the other experiments.

\section{Indirect measurements}

The indirect methods in nuclear astrophysics (IMNA) are those methods in which we make studies using nuclear reactions at much higher energies than those available/important in the stars, at typical nuclear laboratory energies that are 100-1000 times larger. We seek and obtain in these measurements information which is later used to evaluate reaction rates at very low energies, relevant for nuclear astrophysics. Experimentally, there are advantages related firstly to the larger cross sections of the reactions employed and secondly to the fact that by using Radioactive Ion Beams (RIB), available lately in several laboratories, one can extend our measurements to stellar reactions which involve unstable 
partner(s). These reactions are overwhelmingly the most numerous in stellar processes. So far direct astrophysical measurements with RIB are not possible (but attempts and progress are made in this direction). In IFIN-HH we do not have RIBs, therefore this type of measurements we can only pursue at outside facilities. (In parenthesis: these involve proposals and PAC approval and may take a lot of time. A bonus is that they imply collaborations, sometimes large collaborations!) Important to notice in IMNA is that good, reliable theoretical support is crucial; I will briefly argue on this at the end of the paper.

NAG was involved in many experiments and in some theory subjects in the last years. I will mention only those where we had a leading role, or at least an important one.

\subsection{Breakup of loosely bound radioactive nuclei}

I was talking about this breakup subject several times. The basic idea is that for loosely bound projectiles in both proton Coulomb and nuclear breakup $\mathrm{X} \rightarrow \mathrm{Y}+\mathrm{p}$ reactions information can be obtained that is directly relevant to radiative proton capture reactions $\mathrm{Y}(\mathrm{p}, \gamma) \mathrm{X}$ in stars. We were proposing and/or preparing experiments on this subject, this time we carried a long awaited ${ }^{9} \mathrm{C}$ Coulomb and nuclear breakup experiment at RIBF of RIKEN in Wako, Japan. The experiment was carried out at the SAMURAI spectrometer using a secondary ${ }^{9} \mathrm{C}$ beam at $160 \mathrm{MeV} /$ nucleon. It was the first in a series of four proposed (and PAC accepted) experiments with proton-rich radioactive beams at RIBF, the HI-p collaboration. This was a first at a facility that has so far excelled in producing neutron-rich secondary beams. Moreover, it was notable that a neutron-rich primary beam, ${ }^{18} \mathrm{O}$ at 230 $\mathrm{MeV} /$ nucleon was used to produce a very proton-rich ${ }^{9} \mathrm{C}$ secondary beam. Our group has worked with the RIBF group to obtain a reasonably intense and clean beam: ${ }^{9} \mathrm{C} 87 \%$, plus ${ }^{8} \mathrm{~B}$ and ${ }^{7} \mathrm{Be}$ impurities. Inclusive and exclusive measurements at energies around 160 $\mathrm{AMeV}$ for ${ }^{9} \mathrm{C}$ were carried out in order to evaluate the astrophysical $S_{18}$ factor for the inverse process ${ }^{8} \mathrm{~B}(\mathrm{p}, \gamma){ }^{9} \mathrm{C}$ at energies in the region of astrophysical interest. This radiative proton capture on ${ }^{8} B$ is important in the hot $p p$ chains, in explosive Hydrogen burning ( $p p I V$ and $\operatorname{rap} I$ ), at temperatures between $0.05<T_{9}<1 \mathrm{~K}$, as possible alternative paths across the $\mathrm{A}=8$ mass gap. Another goal of this experiment was a detailed study of the breakup reaction mechanism. A $\mathrm{C}$ target was used for the nuclear breakup and a $\mathrm{Pb}$ target for the Coulomb dissociation study. The data analysis is not finished at this time, a preliminary report was presented in Dec. 2018 at the Nucleus-Nucleus Collisions 2018 conference, Saitama, Japan and a publication was accepted [8]. It reports in particular about the design and functioning of a Si detector system and its attached electronics used in front of SAMURAI spectrometer, right after the target.

\subsection{Spectroscopy of resonances}

In many reactions in addition to a continuous component of the astrophysical S-factor, low energy resonances play a role, sometimes a decisive role. To determine their position $\left(\mathrm{E}_{\mathrm{res}}\right)$ and their resonance strength any spectroscopic method is valid. We worked on two types of experiments.

One involves beta-delayed proton decay $(\beta p)$ of exotic nuclei. Started by the author at Texas A\&M University a few years back, we advanced in instrumentation in the last few years and we had the first experiments. It works like this: the low energy resonances that contribute to the stellar reaction rates for proton radiative captures can, in cases where energy and selection rules permit, be populated through beta-decay. Then, these states do proton decay. However, the proton energies will be very small for the most important resonances and these energies cannot be easily measured. We designed a method and 
detectors to reach down at energies as low as $E_{p}=100 \mathrm{keV}$ without being affected by the always present and overwhelming $\beta$ background. It is based on a gas detector working in ionizing chamber regime and a special amplifying device called micromegas, a region of space between two electrodes separated by 256 microns that works in an avalanche regime that delivers good amplification of the original signal by up to $10^{4}$, while keeping a very good resolution. Two versions of the detector called ASTROBOX [9] and ASTROBOX2 [10] were built. A local version ASTROBOX2E was put together in our group. I send you to these publications for details. The $\beta$ p-decay of ${ }^{23} \mathrm{Al},{ }^{31} \mathrm{Cl}$ and ${ }^{35} \mathrm{~K}$ were measured and we plan soon to measure ${ }^{27} \mathrm{P}$, all at Texas A\&M University with exotic beams produced and separated by the superconducting cyclotron K500 and the mass separator MARS.

Another type of experiments was one of gamma-ray spectrometry. We have measured the reaction ${ }^{28} \mathrm{Si}(\alpha, \mathrm{n} \gamma)^{31} \mathrm{~S}$ at the $9 \mathrm{MV}$ tandem of IFIN-HH using the ROSPHERE detector array with neutron detectors added. This analysis is also not finished yet and I will not further describe it here.

\section{Theory for nuclear astrophysics}

I will only state here (as I did in the presentation at ENNAS 2019) that there are groups/persons at IFIN-HH working on three different topics of importance in nuclear astrophysics. These are:

- support for IMNA: we have a long-term program to understand and describe reactions between nuclei, including those with RIB. For this purpose, we work with prof. F. Carstoiu on the problem of optical model potentials [11];

- the evaluation of the contribution of excited states in reactions and processes that take place in hot stellar plasma - prof. Alexandrina Petrovici and her group [12];

- baryonic equation of state under extreme conditions - prof. Adriana Raduta [13].

\section{Conclusions}

I could sketch here the few directions of work and progress in nuclear astrophysics at IFINHH Bucharest-Magurele. I insisted on experiments and only mentioned briefly theory. I should mention too that essentially the same NAG group initiated and organized a few events in the field, events that you may have taken part in. One was the training school (a hands-on experiment) for the COST action CA16117 ChETEC, one other the Carpathian Summer School of Physics, the sister of ENNAS, attended again by around 100 people in its latest editions (2016 and 2018 [14]), and the third was the ECT* workshop in Trento, Italy, Nov. 5-9 2018 with a title relevant "Indirect Methods in Nuclear Astrophysics" [15].

\section{Acknowledgement}

The author acknowledges the support of the Romanian Ministry of Research and Innovation under grant nr. PN-III-P4-PCE- 2016-0743.

\section{References}

1. L. Trache, Journal of Physics: Conference Series 703, 012011(2016).

2. C.L. Jiang et al., Phys. Rev. C 97 (2018) 012801. doi:10.1103/PhysRevC.97.012801.

3. A. Tumino et al., Nature 557, 687 (2018).

4. D. Tudor et al., arXiv:1907.03596 [physics.acc-ph] and submitted to NIM A, July 2019 
5. G. R. Caughlan, W. A. Fowler, Thermonuclear reaction rates v, Atomic Data and Nuclear Data Tables 40, 283-334 (1988).

6. D. Tudor et al, Journal of Physics: Conference Series 703, 012028 (2016); N.T. Zhang et al., arXiv:1909.07012v1[nucl-ex] and submitted to Phys. Let. B, Sept. 2019.

7. G. Gyurky, et al., Phys. Rev. C 86 (2012) 041601.

8. A.I. Chilug et al., in Proc. NN2019, JPS Conf. Series, accepted, in press;

9. E. Pollacco et al., NIM A 723, 102 (2013).

10. A. Saastamoinen et al., NIM B 376, 357 (2016).

11. F. Carstoiu and L. Trache et al., Phys. Rev. C 85, 054606 (2012); F. Carstoiu et al. Rom. J. Phys. 61, 1180 (2016).

12. A. Petrovici et al., Phys. Rev. C 100, 015810 (2019) and references within.

13. Ad. Raduta et al. MNRAS 487, 2639 (2019) and references therein, https://doi.org/10.1093/mnras/stz1459

14. L. Trache and A. Spiridon, eds, "Exotic Nuclei and Nuclear/Particle Astrophysics (VII). Physics with small accelerators”, Proc. CSSP18, AIP Conf. Proc Series, vol 2076, N. York, 2019.

15. http://www.ectstar.eu/node/4228. 